\section{Blood Ammonia and the Deaminases of Adenosine} and Adenylic Acid

ThE characteristic value of the general blood ammonia in man was shown to be either zero or below the analytical level ${ }^{1}$. This has been confirmed for the rabbit and by $\mathrm{H}$. Kropowski for the dog, using the technique of this laboratory ${ }^{2}$.

After shedding, the ammonia develops from three sources. The first or alpha ammonia (about $40 \gamma$ ammonia $N / 100 \mathrm{ml}$.) forms immediately after shedding whether the $p \mathrm{H}$ of the blood be maintained or not, but does not form in the presence of carbon dioxide. The amino compound involved may be adenosine protected by carbon dioxide (though the complex formation does not appear to be readily reversible) and deriving in turn from one or other of the adenylic acids. Plasma has been shown to deaminate adenosine with great specificity, leaving unattacked more than fifty other substances of biological significance and with amino or volatile amine groups. This plasma deamination of adenosine and its great specificity was shown by one of us $^{3}$ prior to the communication of Drury et al. to the Physiological Society in January 1937.

The second or beta formation (about $1 \mathrm{mgm}$. $\mathrm{N} / 100 \mathrm{ml}$. in the rabbit) comes from adenylpyrophosphate in the red corpuscles, breaking down mainly through the following stages: adenylpyrophosphate $\rightarrow$ adenyldiphosphate +1 phosphate $\rightarrow$ adenosine +2 phosphate $\rightarrow$ inosine +3 phosphate + ammonia. (The phasic formation of ammonia previously reported ${ }^{4}$ appears to be associated with the re-esterification of adenylic acid.) Adenylpyrophosphate disappears practically quantitatively in rabbit blood with the appearance of free ammonia. The chief reason for supposing the final stages is that muscle adenylic acid in high concentration is freely deaminated by laked red corpuscles and vegetable adenylic acid also, but only at a small fraction of this rate. As the concentrations of the acids are diminished so also is the deamination rate, but to a lesser degree for vegetable adenylic acid. At a concentration of 0.05 per cent of the nucleotide they are deaminated at about the same rate. This can be interpreted satisfactorily only on the basis of an initial dephosphation - at the low concentrationsfollowed by a deamination of the formed adenosine.

The third or gamma ammonia (about 100-200 $\gamma$ $N / 100 \mathrm{ml}$. for man, and a mean of about $350 \gamma N / 100$ ml. for the rabbit, judging from the twenty-four hours formation) appears to derive largely if not entirely from vegetable adenylic acid, this being first dephosphated and then deaminated. The following evidence exists for this supposition.

(1) Vegetable adenylic acid is deaminated at the required rate, muscle adenylic acid and adenylpyrophosphate being excluded by the fact that the action of striped muscle extract has no effect in raising the plasma ammonia.

(2) The rate of the gamma ammonia formation in plasma plotted against the $p \mathrm{H}$ shows two peaks, one in the region of 6.8 and the other at about $8 \cdot 6$. This double peak is characteristic of a phosphatase action signifying a preliminary dephosphation. When vegetable adenylic acid is added to plasma, the extra ammonia formed after 24 hours shows a similar double peak and in the same position.

(3) A large number of other possibilities has been excluded; for example, guanylic acid, guanosine, eytydylic, cytidine, ete., but not the adenyl com- pounds of desoxyribose, which remain as possible but unlikely precursors.

Concerning the adenylic acid and adenosine deaminases of blood and tissues, it may be noted that adenylic acid in laked blood (and five other tissues similarly examined) is deaminated in proportion to its concentration beyond a certain critical value of $0 \cdot 1-0 \cdot 2$ per cent of the nucleotide. The activity of the deaminase also per unit volume of blood increases markedly with the dilution. On the other hand, the ferment in striped muscle (about forty times greater in the mean than that of any other tissue) acts independently of the substrate concentration. The indication is that the ferment in the red corpuscle and other tissues - except voluntary muscle -is reversibly bound to some inhibiting substance, which may be described as a protector substance for adenylic acid. Comparative values of the adenosine and adenylic acid deaminases (adenylic acid in 1 per cent concentration) were studied in thirty-six tissues of the rabbit. With regard to the adenosine deaminase, it is a curious fact that the appendix showed the highest concentration in the body, having a mean value of $\mathbf{5 5 . 6}$ units of enzyme concentration (the unit being $\gamma N$ / minute/gm. tissue) compared with about 6-8 for whole blood, ileum and cæcum. The values for duodenum and jejunum were also high but not so high as the appendix. Of the eleven types of glandular tissue studied, it is noteworthy that the liver came second lowest with $3 \cdot 7$ units (it contained the lowest amount of adenylic deaminase, namely, $2 \cdot 2$ units). The muscle group contained the least amounts of adenosine deaminase, the muscle of the auricle being highest in the series with 6.8 units and voluntary muscle lowest with $0 \cdot 9$. The content in adenylic acid deaminase followed to some extent that for adenosine, with many marked differences, the most extreme being voluntary muscle, which contained in the average about 1,000 enzyme units. This enzyme also is greatly in excess of the adenosine deaminase in conducting nerve, cerebral cortex and pituitary gland; and in the alimentary distribution we have the proportion reversed. No pure adenylic acid deaminase was found in plasma.

The above research was supported by a grant from the Irish Medical Research Council.

Department of Physiology
Roward J. Conway.
and Biochemistry,
University College,
Dublin.
Sept. 3.
${ }^{1}$ Conway, E. J., Biochem. J., 29, 2755 (1935).
2 Private communication from the Warsaw laboratory.
${ }^{3}$ Thesis to U.C.D. on ammonia formation in blood and tissues (1936).
4 Conway, E. J., and Cooke, R., NATURE, 139, 627 (1937).

\section{Application of the Feulgen Method to the Study of Viruses}

'Nucleord' bodies giving a positive Feulgen reaction have been shown to be integral constituents of various kinds of spore-bearing bacilli ${ }^{1}$ and also of Bact. Coli and "Bact. paratyphosum"2. Photographed in ultra-violet light, these bodies proved to possess the specific absorption characteristic of nucleic acid.

So far as we are aware, Haagen is the only investigator who has applied this reaction to a virus. He has stated ${ }^{3}$ that the inclusion bodies of vaccinia give 